\title{
Un lieu pour les figures dans la théorie de l'argumentation
}

A Place for Rhetorical Figures in Argumentation Theory

\section{Christian Plantin}

\section{(2) OpenEdition}

1 Journals

\section{Édition électronique}

URL : http://journals.openedition.org/aad/215

DOI : $10.4000 /$ aad. 215

ISSN : 1565-8961

Éditeur

Université de Tel-Aviv

Référence électronique

Christian Plantin, « Un lieu pour les figures dans la théorie de l'argumentation », Argumentation et Analyse du Discours [En ligne], 2 | 2009, mis en ligne le 01 avril 2009, consulté le 23 septembre 2019. URL : http://journals.openedition.org/aad/215; DOI : 10.4000/aad.215

Ce document a été généré automatiquement le 23 septembre 2019.

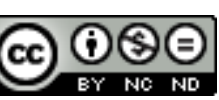

Argumentation \& analyse du discours est mis à disposition selon les termes de la licence Creative Commons Attribution - Pas d'Utilisation Commerciale - Pas de Modification 4.0 International. 


\title{
Un lieu pour les figures dans la théorie de l'argumentation
}

\author{
A Place for Rhetorical Figures in Argumentation Theory
}

\section{Christian Plantin}

1 Cet article traite d'un thème à la fois ennuyeux et fascinant, les figures du discours. N'étant pas particulièrement adepte des errances dans la «sylva rhetoricæ $»^{2}$ et des (re-)labellisations, (re-)définitions, (re-)classifications des centaines de figures qui nous ont été léguées par la tradition rhétorique, je me limiterai, si l'on peut dire, à la question des relations entre les figures du discours ${ }^{3}$ et quelques aspects centraux du discours argumentatif.

2 Conley note que « nothing [he has] seen in the critical literature pays much attention to two important subjects treated by Perelman in the Traité :loci and figures» $(1999: 62)^{4}$. On peut vérifier rapidement cette affirmation en parcourant les actes des trois derniers colloque de l'ISSA (van Eemeren et al. 1999, 2003, 2007) ; l'expérience montre qu'elle est bien vérifiée pour ce qui concerne les figures - concernant les loci, la conclusion serait sans doute différente.

3 L'opposition populaire entre un discours orné-rhétorique et un discours argumentatif remonte au moins à l'école de Ramus, et elle a été fortement réaffirmée par Locke, dans la perspective moderne d'un discours visant à «the preservation and improvement of truth and knowledge » $(\S 1)$. Cette opposition peut être remise en question sur une base étymologique, et, de façon plus cruciale, pour des raisons qui touchent à l'étude linguistique du discours. Pour cela, le $\$ 2$ développe d'abord une direction de recherche inspirée du traitement fondateur que Perelman et Olbrechts-Tyteca proposent pour les figures du discours dans le Traité de l'argumentation (que nous désignerons par les initiales $T A$ ) pour traiter des questions de choix, de présence et de communion. Les figures ne sont pas considérées comme des décorations, mais comme une théorie de la structuration argumentative de la lexis, en d'autres termes, comme des instruments permettant de traiter de façon spécifique la sémantique du discours argumentatif. 


\section{Ornements et arguments}

\subsection{Différentes formes de rhétorique}

4 Certaines définitions de la rhétorique ne font pas de place à la question des figures. Par exemple, Bitzer a redéfini la rhétorique comme la capacité de transformer une situation par le langage - « rhetoric is a mode of altering reality » (Bitzer 1968:250) - ; c'est une définition d'une rhétorique sans ornements ni éloquence ${ }^{5}$.

5 Si l'on considère les conceptions plus traditionnelles de la rhétorique, Kennedy distingue entre deux types de rhétorique, une rhétorique primaire ["primary »], orale, persuasive ; et une rhétorique secondaire [«secondary »], écrite, lettrée artistique, la première étant sans doute plus argumentative, et la seconde plus ornementale.

On oppose également une rhétorique extrovertie [extroverted, extrinsisch] à une rhétorique introvertie [introverted, intrinsisch] (Kienpointner 2003). Cette rhétorique extrovertie est focalisée sur l'interlocuteur, elle est interactive, communicationnelle, éloquente. La rhétorique introvertie est centrée sur le locuteur et sur l'exactitude de l'énonciation; La Bruyère a exprimé le sentiment profond de cette rhétorique qui a renoncé à l'éloquence et à la persuasion : "Il faut chercher seulement à penser et à parler juste, sans vouloir amener les autres à notre goût et à nos sentiments; c'est une trop grande entreprise $»^{6}$. L'une et l'autre peuvent être fortement argumentatives et ornementales. On pourrait distinguer les théories introverties et extroverties de l'argumentation.

7 L'opposition entre une rhétorique des figures et une rhétorique des arguments est évidemment une survivance et une exacerbation de la distinction entre les deux moments fondamentaux de la rhétorique ancienne, l'inventio et l'elocutio ${ }^{7}$. L'inventio est une question de pensée, l'elocutio une question de langage, elle met en mots les données construites par l'inventio, et ordonnées par la dispositio. L'elocutio est caractérisée par quatre qualités, la correction grammaticale (latinitas), la perspicuitas, qui a trait à la clarté du message (pour l'interlocuteur), l'aptum qui souligne la nécessité de l'unité du message et de son adaptation aux circonstances de l'énonciation, et enfin l'ornatus, qui en constitue l'élément essentiel, et qui est le lieu théorique des figures.

8 La rupture entre inventio et elocutio est généralement attribuée à Ramus, qui a réduit la rhétorique ancienne à l'elocutio avec l'actio, alors que l'inventio, la dispositio et la memoria sont réaffectées à la dialectique.

Dans la littérature francophone en rhétorique et argumentation des années 1970, cette opposition entre une rhétorique des figures et une rhétorique des arguments a été revécue, sans référence à Ramus, comme une incommensurabilité entre le Traité de l'Argumentation d'une part et la Rhétorique Générale du Groupe $\mu$ (Dubois et al.) (1970). Cet ouvrage se focalise sur l'analyse linguistique des figures, ne traite pas des effets esthétiques des figures et ne mentionne pas la question des arguments. ${ }^{8}$

\section{2 « Rhetoric, that powerful instrument of error and deceit...»}

De ces distinctions entre diverses rhétoriques, la plus intéressante pour la théorie de l'argumentation est la distinction entre ornement et argument. Les figures et les tropes sont d'abord définies dans le cadre de l'ornatus, puis, par synecdoque, l'elocutio est 
assimilée à l'ornatus, et finalement la rhétorique elle-même à l'elocutio. C'est cette vision ornementale de la rhétorique qu'on a voulu opposer au discours sain et correct de l'argumentation. Le texte suivant de Locke est un locus classicus du discours contre le « langage figuré »:

Since wit and fancy find easier entertainment in the world than dry truth and real knowledge, figurative speeches and allusion in language will hardly be admitted as an imperfection or abuse of it. I confess, in discourses where we seek rather pleasure and delight than information and improvement, such ornaments as are borrowed from them can scarce pass for faults. But yet if we would speak of things as they are, we must allow that all the art of rhetoric, besides order and clearness; all the artificial and figurative application of words eloquence hath invented, are for nothing else but to insinuate wrong ideas, move the passions, and thereby mislead the judgment; and so indeed are perfect cheats: and therefore, however laudable or allowable oratory may render them in harangues and popular addresses, they are certainly, in all discourses that pretend to inform or instruct, wholly to be avoided; and where truth and knowledge are concerned, cannot but be thought a great fault, either of the language or person that makes use of them. What and how various they are will be superfluous here to take notice; the books of rhetoric which abound in the world, will instruct those who want to be informed: only I cannot but observe how little the preservation and improvement of truth and knowledge is the care and concern of mankind; since the arts of fallacy are endowed and preferred. It is evident how much men love to deceive and be deceived, since rhetoric, that powerful instrument of error and deceit, has its established professors, is publicly taught, and has always been in great reputation: and I doubt not but it will be thought great boldness, if not brutality, in me to have said thus much against it. Eloquence, like the fair sex, has too prevailing beauties in it to suffer itself ever to be spoken against. And it is in vain to find fault with those arts of deceiving, wherein men find pleasure to be deceived. (Locke 1690. Book III, ch. X, sect. 34)

Paul de Man (1978) a donné un splendide commentaire de ce passage, où il montre que l'enjeu est ici le statut de la langue naturelle en science et en philosophie: « at times it seems as if Locke would have liked nothing better that to be allowed to forget about language altogether, difficult as it may be in an essay having to do with understanding » (1972, p. 12).

Cette conclusion n'invalide pas directement la thèse de Locke. Il est en effet parfaitement possible de considérer que celle-ci porte sur le langage ordinaire et sur son adéquation en tant que langage de la connaissance. De fait, le langage naturel n'est pas le langage dans lequel « knowledge is preserved and improved». La science laisse effectivement de côté la langue naturelle, puisque ce n'est pas dans ce langage qu'elle se développe mais dans des langages formels et techniques. Néanmoins, de Man a raison quand il insiste sur le caractère contradictoire d'une entreprise qui se propose d'analyser le raisonnement en langue naturelle en condamnant le langage naturel.

On peut schématiser comme suit le discours contre les figures qui les assimile à des ornements pour conclure à leur caractère fallacieux.

Premièrement, dans le cadre du déroulement du discours argumentatif, toute décoration est un divertissement, c'est à dire un distracteur. En conséquence, elle manifeste un défaut de pertinence, elle est ainsi fallacieuse par ignoratio elenchi et question begging.

15 Deuxièmement, le concept classique de discours figuré est fondé sur l'idée de choix possible entre deux chaînes de signifiants pour exprimer le même être, contenu 
sémantique ou état du monde. Ceci présuppose une surabondance des mots par rapport aux modestes, quoique strictes, exigences de l'exposé et du développement de la vérité. L'existence d'au moins deux chaînes de formes expressives est à la source de la fallacie de verbiage. Le choix final favorise systématiquement le rare, l'artistique, le recherché...par rapport à la façon de parler ordinaire, simple et directe ${ }^{9}$. Cette préférence entraîne une rupture entre ce qui est attendu par le locuteur et ce à quoi le confronte le flux du discours. Ses attentes sont frustrées et dépassées. La figure ornementale est décalée, elle introduit une différence et une surprise. Par définition, la surprise suppose une perte de contrôle, elle est le prodrome d'une émotion. Elle ouvre ainsi la porte au vaste ensemble des fallacies ad passiones; ce lien est explicite dans Locke.

Troisièmement, la figure décorative est l'instrument préféré de la fallacie de verbiage, une sorte de méta-fallacie qui ouvre la voie à toutes les autres :

As Richard Whately remarked, "... a very long discussion is one of the most effective veils of Fallacy; ...a Fallacy which when stated barely ... would not deceive a child, may deceive half the world if diluted in a quarto volume" (Elements of Logic, [1844, p. 171]. Consequently, an important weapon against fallacy is condensation, extracting the substance of an argument from a mass of verbiage. But this device too has its dangers; it may produce oversimplification, that is, the fallacy a dicto secundum quid, of dropping relevant qualifications. When we suspect a fallacy, our aim must be to discover exactly what the argument is; and, in general the way to do this is first to pick out its main outlines and then to take into account any relevant subtleties or qualifications (Mackie $1967: 179$ ).

En particulier, le rejet du verbiage entraîne le rejet de l'éloquence fondée sur l'abondance de mots, copia verborum. La Logique de Port-Royal stigmatise la technique de l'inventio, la topique, parce qu'elle stimulerait la «mauvaise fertilité des pensées communes » (Arnauld et Nicole 1965 : 235). La même critique pourrait être adressée aux techniques de l'elocutio qui, en développant l'abondance oratoire, engendrent les congeries, précisément «a mass of verbiage » pléonastique. Le discours rhétorique est sale : il ne sait pas effacer ses brouillons, il érige en figure ses corrections (cf. § 2.3, sur les figures du choix).

Quatrièmement, les figures violentes sciemment trois principes gricéens, elles pèchent contre la quantité, la qualité et la pertinence - pour reprendre le mot de Klinkenberg, elles sont impertinentes. Qui plus est, elles ne respectent pas la règle de non contradiction: la métaphore est à la fois vraie et fausse, coupable d'ambiguïté et d'erreur catégorielle (Groupe $\mu 1970$ : 129-130). Le plaisir qu'on y prend est malin.

Pour garantir l'accès le plus direct aux objets et à leurs liens naturels le langage argumentatif-scientifique doit être réglé, non ambigu, sans défaut ni excès, exactement proportionné à la nature des choses, en d'autres termes, transparent (ad rem, Locke 1972 [1690]). La vérité et l'honnêteté doivent sortir nues du puits; les figures qui prétendent l'orner, en fait la voilent. Les ornements sont pires que les fallacies, ils en sont le masque.

\subsection{Un argument étymologique contre la conception décorative de l' ornatus}

On pourrait se demander si les figures sont réellement des ornements. On pourrait proposer un argument contre une vue décorative de l'ornatus. Le mot ornement, qu'on 
attache aux figures est une copie du substantif latin ornamentum (adjectif ornatus, verbe ornare). Selon le dictionnaire latin-français, le sens premier de ornamentum est: «1. appareil, attirail, équipement [...] harnais, collier [...] armure» (Gaffiot 1934, art. ornamentum). Ce sens fondamental est aussi celui du verbe ornare du participe passé adjectif ornatus. On traduit donc «naves omni genere armorum ornatissimae " (C. Jules César, De bello gallico 3, 14, 2) par: "navires abondamment pourvus de tout l'équipement nécessaire [armes et agrès] " (voir aussi l'article ornatus).

21 Ainsi, un discours bien ornatus est un discours bien équipé pour bien remplir sa fonction. S'il s'agit d'un discours produit dans le cadre d'une esthétique de la décoration ${ }^{10}$, on pourra parler d'un discours bien décoré. Mais, si l'on a affaire à une intervention faite dans le cadre d'un choix à opérer dans les affaires publiques, ce sera un discours bien argumenté ; on n'hésitera pas à dire que les arguments font partie des ornamenta du discours, c'est-à-dire de son équipement. On pourrait considérer le traitement des figures dans le TA comme la meilleure élaboration de cette proposition que nous fait le mot lui-même.

\subsection{Métonymie : un même mécanisme pour des tropes et des topoi}

Nous avons tenté de montrer dans d'autres articles $(1993,2002)$ que les mécanismes qui régissent le fonctionnement d'un " master trope ${ }^{11}$ comme la métonymie n'étaient au fond pas différents de ceux qui permettent de dériver une conclusion d'un argument. Dans la métonymie de l'effet, la désignation d'un effet est remplacée par celui de la cause qui lui est associée. Dans l'argumentation par les conséquences, le jugement de valeur porté sur une conséquence est transféré à sa cause. Les lois qui gèrent ce type de substitution de signifiants pour le trope ne sont pas différentes de celles qui concluent à l'acceptabilité d'une cause à partir de celle de son effet (argumentation par les conséquences). Il faudrait donc parler d'argumentation métonymique, comme on parle de désignation métonymique. On peut proposer d'analyser dans ce sens des argumentations comme «Il n'y a aucun rapport entre des livres et puis des meubles. C'est pour cela que le dimanche, on pourra acheter des livres mais pas la bibliothèque où les ranger " (Publicité, Le Monde, 18 septembre 1993) - où le caractère rationnel de l'appel à l'ouverture des magasins de meubles le dimanche est métonymiquement lié à la donnée "les librairies peuvent ouvrir le dimanche ». Elle est également très visible dans le cas des argumentations sur la personne et ses actes (Perelman 1952). L'avocat de Paul Touvier, qui avait fui la justice pendant 25 ans, et a finalement été condamné pour crime contre l'humanité, peut ainsi argumenter que «la conduite de Touvier depuis 25 ans est admirable » : c'est dire métonymiquement que Touvier est admirable. De même, on ne peut juger «l'éducation que Touvier a donnée à ses enfants " sans juger Touvier, et admirer cette éducation, c'est admirer Touvier, par une métonymie argumentative substituant le producteur au produit, variante de la métonymie de l'effet (Plantin 1993 et 2002). La métonymie transfère très bien les bonnes raisons. La production de la conclusion raisonnable n'est pas différente de celle de la décoration séduisante ; la raison est figurée et la figure raisonnable. 


\section{Les figures dans le $T A$}

\subsection{Soixante-quatorze étiquettes pour les figures}

Opposant la vision perelmanienne de l'argumentation à la «renegade logic»de Toulmin - qualificatif à la fois désobligeant et injuste - Conley observe que le $T A$ propose « more than eighty different modes of argument and illuminating observations on the argumentative function of more than sixty-five different figures» (Conley 1984 : 180-181). Soulignons d'abord que, dans la mesure où le TA se veut être une « Nouvelle rhétorique ", cette constatation n'a rien de surprenant, l'intérêt pour les figures étant une caractéristique de la rhétorique ancienne, rhétorique intégrale jusqu'à Ramus, comme nous l'avons noté.

L'index du TA distingue deux entrées, d'une part «figures traditionnelles » et d'autre part « figures ", dont les subdivisions renvoient à l'argumentation. L'index mentionne en outre quelques autres cas isolés, comme l'accumulation, qui ont été ajoutés à la liste ci-dessous. Globalement, on trouve donc les étiquettes suivantes de figures et de tropes. 12

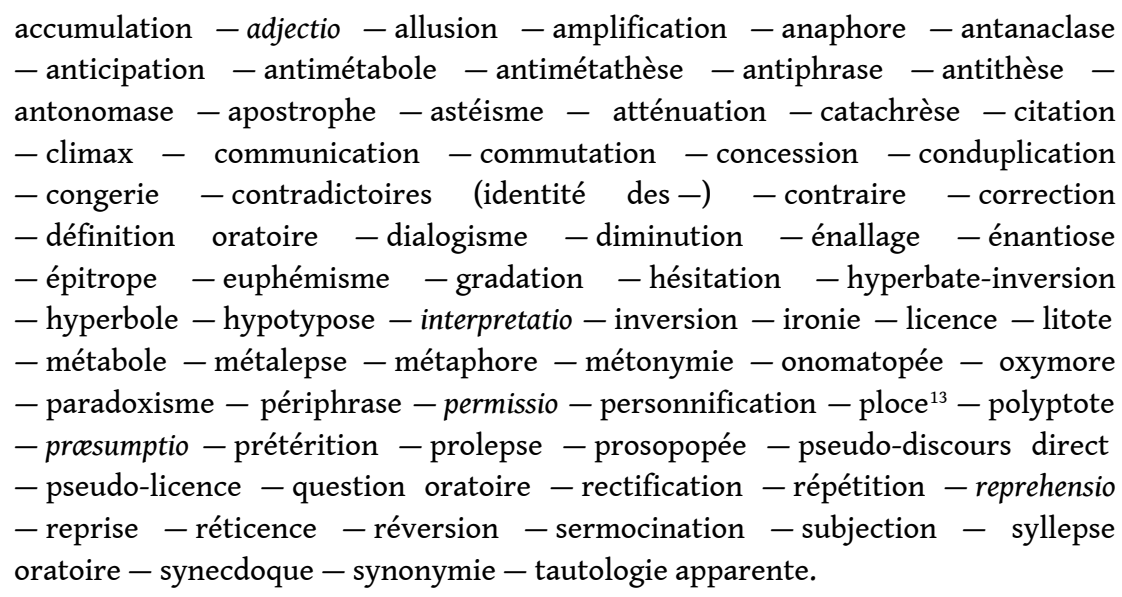

Du point de vue de l'argumentation, on n'a pas à donner de définition de ce qu'est une figure autre que ce genre de collection: est figure ce que les traités de rhétorique appellent figure. Le but est précisément de les (ré)intégrer dans le cadre du discours argumentatif.

\subsection{Une théorie des figures ${ }^{14}$}

En outre, le TA propose une théorie des figures que nous allons d'abord discuter. Elle est exposée au $\$ 41$ « Figures de rhétorique et d'argumentation »:

Nous considèrerons une figure comme argumentative si, entraînant un changement de perspective, son emploi paraît normal par rapport à la situation suggérée. Si, par contre, le discours n'entraîne pas l'adhésion de l'auditeur à cette forme argumentative, la figure sera perçue comme ornement, comme figure de style. Elle pourra susciter l'admiration, mais sur le plan esthétique, ou comme témoignage de l'originalité de l'orateur.

On voit, dès lors, qu'on ne saurait décider, d'avance, si une structure déterminée doit être considérée ou non comme une figure, ni si elle jouera le rôle de figure argumentative ou de figure de style; tout au plus peut-on déceler un nombre de structures aptes à devenir figure (229) 
passage est énigmatique, et, comme Parson et Ziegelmüller (2003: 813), on peut avoir quelques difficultés à en saisir le sens profond. Admettons que, en tant qu'elle est dotée d'une "structure déterminée", une figure est un exemple de "technique discursive ». D'après la définition de l'argumentation proposée dans le $T A$, « l'objet de [la théorie de l'argumentation] est l'étude des techniques discursives permettant de provoquer ou d'accroître l'adhésion des esprits aux thèses qu'on présente à leur assentiment » (1970 [1958] : 5), un point c'est tout. Rien n'est dit sur une possible différenciation des techniques discursives selon que leur usage entraîne ou non l'adhésion de l'interlocuteur dans un cas précis; un usage malheureux d'un topos argumentatif ne produit pas un topos "décoratif ». La «technique discursive " reste argumentative, et rien ne permet de penser qu'il en va autrement pour la «structure déterminée » que constitue la figure argumentative.

Si on fait dépendre la qualité, argumentative ou ornementale, d'une figure de sa réception effective, et le TA traite pas de la réception réelle, on reste court. Normalement, on évite l'impasse en utilisant cette merveilleuse manœuvre aristotélicienne qui consiste à introduire de l'indirection dans le procès de « persuasion » : la rhétorique s'intéresse non pas aux inducteurs effectifs de persuasion, mais à ce qui est «potentiellement persuasif » (Rhét. I, 2, 1355b25-30; p. 124); elle construit des conclusions plausibles non pas pour un individu donné, mais pour «telle sorte de gens » (Rhét. I, $2: 1356$ b30 ; p. 130) ${ }^{15}$. En conséquence, si le truc de la persuasion ne marche pas, on ne se heurte à aucun problème théorique, puisque ce qui est en jeu n'est pas l'effet réel sur le récepteur mais simplement l'intention présumée du locuteur visant un auditoire construit par le discours. Le plus prudent est sans doute de renoncer à résoudre l'énigme, puisque le TA lui-même n'explore pas davantage cette orientation. On abandonnera donc cette perspective fonctionnelle qui fait qu'une construction est ornementale ou argumentative selon qu'elle est persuasive ou non pour un auditoire particulier. Elle revient à dire que le récepteur qualifiera d'ornemental tout discours avec lequel il n'est pas d'accord, sans reconnaître une quelconque valeur à ses arguments.

Une autre direction de recherche est suggérée par l'observation que le TA s'intéresse aux figures dans la mesure où elles contribuent de façon systématique à la construction des arguments: c'est un principe que nous adopterons pleinement. Parson et Ziegelmüller (2003) rappellent que la question des figures est celle de la lexis, en d'autres termes que la question des figures repose celle du langage dans l'argumentation.

30 Nous avons vu qu'une vertu classique de l'elocutio est la correction grammaticale. Mais on sait que, pour la rhétorique classique, l'argumentation n'est pas dans la langue, mais dans le discours. Il ne suffit pas de parler, même très correctement, pour argumenter. L'argumentativité vient au discours au terme d'un travail rhétorique, long et difficile pour beaucoup, qui est un travail spécifiquement discursif. C'est dans le cadre de ce travail argumentatif de construction du discours que se pose la question des figures.

31 Les topoi ne tombent pas du ciel dans les enthymèmes, et le but de l'analyse argumentative n'est pas de les y faire remonter. On peut proposer de les considérer comme des «discours pivots" (Plantin 2002) à partir desquels sont produits les discours argumentatifs, par leur actualisation, adaptation, transformation, reformulation... tout cela constituant le travail conceptualisé comme figural par le TA. 
Après la discussion générale des figures au $\S 41$ «Figures de rhétorique et argumentation», le $\S 42$ est consacré aux «Figures du choix de la présence et de la communion » (TA 232-241). Ce paragraphe traite 30 étiquettes, pour en gros 15 types de figures.

\subsection{Le cas des « figures du choix de la présence et de la communion » (TA 232-241)}

Ce serait donc une erreur méthodologique de tenir l'analyse argumentative dans la seule identification des épisodes topiques. Si l'on accepte d'inverser l'image, on dira que les topoi sont les vraies fleurs de rhétorique, et que les figures seraient plutôt l'herbe du pré écologique où viennent les fleurs, ou même la terre qui les nourrit. Mais peut-être appelons-nous herbe ce qui est la plante s'apprêtant à produire la fleur.

c'est parce qu'on ne les cherche pas là où elles sont. On peut faire l'hypothèse que le TA fait appel aux figures précisément lorsque ses auteurs ont besoin de quelque chose que nous appellerions maintenant une « sémantique du discours argumentatif ». Dans le TA, le recours aux figures est une théorie linguistique du discours. Cette perspective rendrait par ailleurs possible une nouvelle vision de l'articulation du TA aux théories généralisées de l'argumentation proposées ultérieurement par Grize et Ducrot, théories qui mettent la problématique globale du discours au premier plan de la réflexion sur l'argumentation, apportant ainsi de nouvelles solutions à un très vieux problème.

figures du point de vue de l'argumentation est le rejet des typologies existantes : « Les classifications des figures généralement utilisées ne peuvent en rien nous aider » (TA 232). Une seconde conséquence est l'inversion radicale de la stratégie d'analyse, en choisissant d'affirmer la prééminence des catégories argumentatives sur les catégories traditionnelles «nous nous demanderons, à propos de tel ou tel procédé ou schème argumentatif, si certaines figures sont de nature à remplir la fonction que nous avons reconnue à ce procédé, si elles peuvent être considérées comme des manifestations de celui-ci »(ibid.). Ce qui revient à une incitation au démantèlement des ensembles constitués de figures : «[...] les figures seront démembrées » (ibid.) et, peut-on ajouter, à leur restructuration. Deux précisions sont nécessaires avant de passer à cette tâche.

de son rôle argumentatif possible ${ }^{16}$. De même, on ne tentera pas de discuter en détail la cohérence hypothétique du traitement des figures dans le TA. Certaines sont traitées au passage, de manière très allusive, et, surtout, on pourrait en ajouter en masse, comme on peut le constater sur le cas des figures d'émotion, ou sur les figures du dialogisme (voir infra 3).

pour construire les concepts argumentatifs de "choix, présence et communion ", afin d'élaborer l'idée que les figures doivent se comprendre comme des opérations de sémantique discursive. 


\subsubsection{Choix}

38 A ce point du $T A$, le concept de choix a déjà été défini comme un trait caractéristique de la situation argumentative : l'argumentation suppose un " choix », un « choix délibéré » (TA 61), entre des possibilités également raisonnables.

39 Le paragraphe 42 se situe dans la seconde partie du $T A$, «Le point de départ de l'argumentation", donc dans un contexte tout différent où il ne s'agit plus des conclusions, mais des données. Il vient après le chapitre 2 , «Le choix des données et leur adaptation en vue de l'argumentation ». Par choix on entend ici le procès de sélection des données pertinentes qui seront retenues pour servir de prémisses à l'argumentation (\$29, p. 154-155).

On peut souligner au passage l'intérêt que présente cette problématique par rapport à une vision (que Perelman considérerait sans doute comme "cartésienne ») faisant comme si les "données» étaient fournies par l'évidence sensible ou posées par l'évidence intellectuelle et non pas construites discursivement. Ici, le choix est effectué entre les données disponibles, afin de constituer les arguments (la confusion entre donnée et argument est ainsi évitée). L'argumentateur choisit l'ensemble des données qu'il considère comme pertinentes pour une question particulière, ou il circonscrit l'information nécessaire pour argumenter sur un thème. Ce choix, parmi les données qui peuvent conduire à la conclusion désirée, s'effectue évidemment au risque d'une petitio principii. ${ }^{17}$ Les figures du choix sont les suivantes :

interpretatio - définition oratoire - périphrase, dont « beaucoup peuvent s'analyser en termes de figures telles la synecdoque... la métonymie... l'antonomase » (TA, p. 234-235) - prolepse ou anticipation (prcesumptio) - hésitation (reprise, reprehensio) ${ }^{18}$, correction (TA 233-235).

41 L'énumération pourrait paraître hétéroclite si on y cherchait une théorie propre de ces figures, mais nous avons vu que le TA rejette explicitement cette intention (voir 2.2). Ces figures introduisent l'idée que le travail de choix s'opère dès le niveau de la désignation des êtres ou des objets ; le TA les présente, très brièvement, en les orientant vers la recherche des modes argumentatifs de désignation. Le TA n'hésite pas à mettre ainsi au premier plan un trait qui serait jugé secondaire dans une théorie autonome des figures. La prolepse est par exemple mentionnée dans la mesure où la manœuvre d'anticipation sur une désignation possible de l'objet par l'adversaire correspond à une stratégie permettant à l'argumentateur de gérer polyphoniquement les multiples désignations argumentatives, sans qu'il y ait recherche d'une "théorie unifiée de la prolepse ».

42 Le concept de choix capte tout ce qui permet de dépasser la désignation "objective ", vers une désignation "orientée ». Il s'agit bien d'intégrer du point de vue non seulement dans l'argument, mais dans l'être même à propos de qui on argumente, via son mode de désignation. Cette vision doit être confrontée en premier lieu aux concepts proposés par la logique naturelle de Grize, qui parle de la saisie des éléments $\mathrm{du}$ réel sous un certain "éclairage " dans le cadre de sa théorie des schématisations (Grize 1990 ; Sitri 2003).

\subsubsection{Présence}

Les données sélectionnées sont dotées, ipso facto, de présence: «un pareil choix accorde à ces éléments une présence, qui est un facteur essentiel de l'argumentation » 
(TA 155). La notion de présence est introduite, d'un point de vue philosophique et psychologique, au $\$ 29$. Dans le discours, elle est produite ou renforcée par un ensemble spécifique de figures qui «[rendent] présent à la conscience l'objet du discours » (TA 235) :

onomatopée - répétition, anaphore - amplification, congerie - conduplicatio, adjectio - synonymie ou métabole - interpretatio ${ }^{19}$ - pseudo-discours direct... sermocination [et] dialogisme - hypotypose, énallage du temps (TA 235239)

L'effet de présence est produit par des mécanismes de grossissement, ou d'effet de transparence du langage: onomatopée, pseudo-discours direct, hypotypose, figures auxquelles on prête le pouvoir de faire halluciner le réel. L'ensemble pourrait être mis en relation avec les études sur les mécanismes de focalisation.

\subsubsection{Communion}

Le concept de communion apparaît dans le $T A$ à la section consacrée au genre épidictique ( $\$ 12$ «Education et propagande») : «dans l'épidictique la communion autour des valeurs est une fin que l'on poursuit, indépendamment des circonstances précises dans lesquelles cette communion sera mise à l'épreuve»(69). Cette communion renforce l'appartenance au groupe et à ses valeurs. Les figures de communion sont les suivantes :

allusion - citation - apostrophe, question oratoire, communication oratoire -

énallage de la personne et du nombre (TA 239-241).

La communion est le stade ultime où s'accomplit la " persuasion ", par la fusion entre l'orateur et son auditoire autour d'un objet. Une telle atmosphère évoque indéniablement une célébration religieuse, et la messe serait en ce sens l'objet sinon rhétorique du moins épidictique fondamental. Quoi qu'il en soit, on voit que les figures de communion correspondent à des formes de mise en scène du dialogue dans le monologue. L'étude empirique des rapports de l'orateur avec son auditoire a évidemment été renouvelée dans le cadre des travaux sur l'interaction d'estrade.

Le paragraphe 42 se conclut comme suit: "Ces quelques indications sur le rôle de certaines figures dans la présentation des données, suffisent, pensons-nous, à montrer comment on peut rattacher leurs effets à des facteurs assez généraux de persuasion " (TA 241). C'est une conclusion cavalière pour un paragraphe très stimulant ; on peut en conclure que les figures du discours, dont l'étude peut paraître dispersée au fil du TA, prend son sens en fonction d'une idée centrale qui, dans le jargon contemporain, peut s'exprimer ainsi: des groupes de figures sont redéfinies comme des stratégies de discours, qui ont tout à voir avec le mode de construction argumentatif des objets et rien à voir avec du décoratif. Elles tendent à définir une sémantique stratégique pour le discours argumentatif, et caractérisent le fonctionnement stratégique du langage.

\section{Les figures comme éléments d'une sémantique du discours argumentatif}

Récapitulons. Nous avons rappelé au § 1 que la question des figures du discours est centrale dans la construction de l'opposition radicale traditionnelle entre le discours argumentatif sain et le discours argumentatif fallacieux, c'est-à-dire rhétorique- 
figuratif. Selon cette façon de voir, dans la perspective de Locke, une première conclusion serait qu'un "Traité de l'argumentation " ne peut en aucun cas être une «Rhétorique », fût-elle « Nouvelle»; c'est toute l'entreprise de Perelman et OlbrechtTyteca, telle qu'elle est résumée dans le double titre de leur ouvrage, qui serait condamnée comme oxymorique. L'importance qu'ils accordent aux figures du discours pourrait ainsi être invoquée comme un argument renforçant cette conclusion.

A cela, on peut répondre deux choses. La première défense, dans le style déconstructiviste de de Man, consiste à montrer que tout le nerf de l'argument de Locke est lui-même suprêmement rhétorique, et qu'il n'y a là rien d'autre qu'une nouvelle et solennelle condamnation rhétorique de la rhétorique, et nous restons toujours aussi incertains sur le statut argumentatif réel de la rhétorique et des figures. En second lieu, comme tente de le montrer le paragraphe précédent, la lecture du TA oriente vers une appréciation plus stimulante des figures, comme des premiers outils commodes et relativement précis pour traiter la question de l'elocutio ou de la lexis. L'argumentation est nécessairement coulée dans un langage, et l'argumentation ordinaire dans le langage ordinaire. La théorie de l'argumentation est nécessairement une forme de théorie du langage et, plus précisément, une théorie du discours: la théorie rhétorique des figures fait partie de la théorie classique du discours argumentatif, elle n'en est pas le « supplément » fallacieux.

Les figures peuvent être abordées et classées à partir de principes très différents : les critères mis en avant par Lausberg (1960), Kienpointner (1999) ou Dubois et al. (1970), Bonhomme (1998) ou Dupriez (1984) - qui opte pour l'ordre alphabétique -, sont différents. La perspective argumentative, qui ne prétend pas plus que les autres à fournir une classification définitive, fournit néanmoins une série propre de critères de reclassement des figures. Il est dans ce sens possible de reconsidérer les figures dans leurs relations avec les opérations argumentatives majeures. Les remarques qui suivent présentent brièvement les deux autres perspectives sous lesquelles on peut intégrer une pensée des figures : la construction discursive des émotions et la représentation de l'opposition fondatrice du dialogue argumentatif dans le monologue argumentatif.

51 La question des figures et des émotions est traitée latéralement dans le TA (Plantin 2004, 2006), principalement au $\S 96$, «La Rhétorique comme procédé ». Les émotions sont considérées dans le procès de construction de l'identité rhétorique du locuteur ${ }^{20}$. En d'autres termes, l'état émotionnel du locuteur peut être inféré à partir de traits de son élocution, comme l'hésitation, l'hyperbate (inversion), défauts de liaison, mélange des figures, considérées ici comme des troubles du comportement attestant une passion; licence, pseudo-licence ou astéisme en d'autres termes, ces figures doivent être considérées comme des déclencheurs d'inférence semi-conscientes sur l'état du locuteur, sollicitant donc l'empathie du public.

Cette liste n'est qu'une ébauche du vaste ensemble hétéroclite de figures fortement inductrices d'émotions. Lausberg considère que les figures suivantes sont " essentiellement émotionnelles": exclamatio, evidentia, sermocinatio, fictio personee, expolitio, similitudo et aversio (1960: $§ 808-851)$. En outre, ces figures doivent être mises en relation avec les règles plus générales de construction des émotions qu'il propose aux $\$ 257.3$. Dans des travaux ultérieurs, nous nous sommes proposé d'élaborer ce point, pour montrer que les émotions sont une composante inhérente au discours argumentatif. Les points de vue sont inséparablement construits émotionnellement et cognitivement; il y a un sens à parler aussi bien de l'argumentation dans l'émotion, que 
de l'argumentation des émotions, cas particulier de la construction discursive des émotions ${ }^{21}$.

Un vaste ensemble de figures permet de conceptualiser la reconstruction monologale des phénomènes interactionnels. Cette vision a été systématisée et généralisée par Bakhtine. Le TA propose quelques aperçus sur la manière dont le monologue intègre le dialogue lorsqu'il traite, comme nous l'avons vu, des questions de la « communion », ou qu'il mentionne des figures dites «quasi-logiques»comme par exemple l'antimétathèse, l'antimétabole, la commutation, le paradoxe, l'oxymore, ou encore l'antanaclase (TA 292-94, 588-90). En suivant cette suggestion, on peut dégager un ensemble riche et cohérent de figures associées à l'émergence puis à l'élaboration de l'opposition discursive qui structure la situation argumentative. Cet ensemble peut être structuré en plusieurs niveaux correspondant aux divers stades de développement de cette situation, depuis l'énantiose jusqu'à la question, puis les différentes stratégies permettant de détruire, désorienter, réorienter ou réfuter le discours de l'opposant. Cet ensemble comprend au moins, par ordre alphabétique, l'adynaton, l'antanaclase, l'antéoccupation, l'antimétabole, l'antiparastase, l'antithèse, l'apodioxis, l'astéisme, le distinguo, la dubitation, l'énantiose, l'épitrope, l'euphémisme, l'interrogation, l'ironie, l'inversion, l'opposition, l'oxymore, la paradiastole, le paradoxe, la prolepse et la subjection (Plantin 2008).

\section{Conclusions}

Dans la mesure où le langage naturel joue un rôle dans l'argumentation ordinaire, Locke a tort et de Man a raison : l'opposition entre argument et ornement est intenable. Conley insiste à juste titre sur le fait que les figures peuvent, et doivent être systématiquement intégrées à la théorie de l'argumentation. A sa manière parfois elliptique, le TA ouvre de nouvelles perspectives, à la fois au niveau des objets et de la méthode. Les figures, véhiculées par une riche, même si parfois chaotique, tradition offrent un excellent angle d'attaque permettant d'approcher la question du langage dans l'argumentation.

Les figures s'analysent comme une théorisation de la notion de stratégie discursive. La plupart des observations faites sur les figures peuvent s'intégrer à des modules ${ }^{22}$ plus larges d'analyse du discours. Les modules émotionnel, objectal, interactionneldialogiques, fournissent de premiers pôles intégrateurs robustes. Ces trois modules ne sont pas les seuls : on pourrait penser, ou rêver, à un module mémoire qui traiterait des facilitateurs de mémorisation; la persuasion se concrétise dans la répétition de formes linguistiques, bons mots et mots à la mode, buzzwords, soundbites et slogans. On peut supposer que des figures de son, de rythme, de parallélisme y contribuent, sans pour autant en être les seuls constituants. Ces modules sont structurés selon des principes spécifiques; ils rendent compte des figures et ils peuvent produire de nouvelles configurations « figurales».

Dans leur contexte rhétorique originel, les figures sont un constituant primordial du discours argumentatif. Si nous considérons positivement le travail des centaines et des milliers de chercheurs qui, ces cinquante dernières années, ont travaillé et qui travaillent à travers le monde sur les interactions langagières, le discours et l'argumentation, alors la théorie des figures doit être repensée en fonction de ces nouvelles perspectives. Cette nécessité n'implique aucun reniement des visions 
fondamentales du TA qui, malgré son titre imposant, ne doit pas être considéré comme une Summa Argumentationis fermée mais comme un pilier du monde en expansion des études d'argumentation.

\section{BIBLIOGRAPHIE}

Aristotle [2005]. Poetics and Rhetoric. Introd. and notes by Eugene Garver, Rhetoric transl. by W. Rhys Roberts.1924, Poetics, transl. by S. H. Butcher. 1911 (New York : Barnes and Nobles)

Arnauld, Antoine \& Pierre Nicole. 1965 [1662]. La logique ou l'art de penser, contenant, outre les règles communes, plusieurs observations nouvelles, propres à former le jugement. Ed. crit. P. Clair et F. Girbal (Paris : PUF)

Bitzer, Lloyd F., 1974 [1968]. « The Rhetorical situation », Fisher, W. R. (ed.). Rhetoric : A tradition in transition (East Lansing : Michigan State University Press), 247-260

Bonhomme, Marc. 1998. Les figures clés du discours (Paris : Seuil, « Mémo »)

Burke, Kenneth. 1969 [1945]. « Four Master Tropes », A Grammar of Motives (Berkeley : University of California Press), 503-517

Conley, Tomas M. 1999. « The Renaissance roots of Perelman's Rhetoric », Eemeren, Frans H. van. 1999, 101-102

de Man, Paul. 1978. "The epistemology of metaphor », Sacks, Sheldon (ed.) 1978. On metaphor (Chicago, University of Chicago Press), 11-28

Dupriez, Bernard. 1984. Gradus. Les procédés littéraires - Dictionnaire (Paris : UGE)

Dyck, Edward F. 2003. « Topos in Rhetorical Argumentation: From Enthymeme to Figure ", Eemeren, Frans H. van et al. (eds), 261-264

Eemeren, Frans H. van, Rob Grootendorst et al. (eds). 1987. Proceedings of the Conference on Argumentation 1986 (Dordrecht : Foris)

Eemeren, Frans H. van, Rob Grootendorst et al. (eds). 1999. Proceedings of the Fourth International Conference of the International Society for the Study of Argumentation (Amsterdam : SIC SAT)

Eemeren, Frans H. van, J. Anthony Blair et al. (eds). 2003. Proceedings of the Fifth International Conference of the International Society for the Study of Argumentation (Amsterdam : SIC SAT)

Eemeren, Frans H. van, J. Anthony Blair et al. (eds). 2007. Proceedings of the Sixth International Conference of the International Society for the Study of Argumentation (Amsterdam : SIC SAT)

Gaffiot, Félix 1934. Dictionnaire illustré latin-français (Paris : Hachette)

Grize, Jean-Blaise. 1990. Logique et langage (Paris-Gap : Ophrys)

Groupe $\mu$ (= Dubois, Jacques, Francis Edeline et al.). 1970. Rhétorique générale (Paris : Larousse)

Kennedy, George A. 1999 [1980]. Classical Rhetoric and Its Christian and Secular Tradition from Ancient to Modern Times (Chapel Hill : University of North Carolina Press) 
Kienpointner, Manfred. 1999. «Figures of speech: Definition, description and critical evaluation », Eemeren, Frans H. van et al. (eds). Proceedings of the Fourth International Conference of the International Society for the Study of Argumentation (Amsterdam : SIC SAT), 445-454

Kienpointner, Manfred. 2003. « Nouvelle Rhétorique / Neue Rhetorik », Ueding, Gerd (ed.). Historisches Wörterbuch der Rhetorik, VI (Tübingen: Niemeyer), 561-587

Klinkenberg Jean-Marie. 1990. « Rhétorique de l'argumentation et rhétorique des figures », Meyer, Michel et Alain Lempereur (éds), 115-137

Klinkenberg, Jean-Marie. 2001. « Retórica de la argumentación y retórica de las figuras: ¿hermanas o enemigas? », Revista electrónica de estudios filológicos 1. http://www.um.es/ tonosdigital/znum1/estudios/Klinkenberg.htm

Kraus, Manfred. 2007. « From Figure to Argument: contrarium in Roman rhetoric ». In Eemeren, F. $\mathrm{H}$. van et al. (eds). Proceedings of the Sixth International Conference of the International Society for the Study of Argumentation (Amsterdam : SIC SAT), 815-822

Lausberg, Heinrich. 1960, Handbuch der literarischen Rhetorik (Munich : Max Hueber)

Lempereur, Alain. 1990. « Les restrictions des deux néo-rhétoriques », Meyer, Michel et Alain Lempereur (éds). 1990. Figures et conflits rhétoriques (Bruxelles : Editions de l'Université de Bruxelles), 139-158

Levinas, Emmanuel. 1987. « Langage quotidien et rhétorique sans éloquence », Hors sujet (SaintClément : Fata Morgana), 201-211 (article publié en 1981)

Locke, John. 1959 [1690]. An essay concerning human understanding I-II. Collated by A. C. Fraser (New York : Dover)

Mackie, J. L. 1967. « Fallacies », Edwards, Paul (ed.). The Encylopedia of Philosophy 3, 169-179

Parson, Donn W. \& George Ziegelmüller. 2003. «Linguistically sound Arguments : Part II. Eloquence and Argument ", Eemeren, Frans H. van et al. (eds). Proceedings of the Fifth International Conference of the International Society for the Study of Argumentation (Amsterdam : SIC SAT), 811-814

Perelman, Chaim. 1952. « Acte et personne dans l'argumentation », Rhétorique et philosophie (Paris : PUF), 49-84

Perelman, Chaim \& Lucie Olbrechts-Tyteka. 1992 (5éd.). Traité de l'Argumentation. La nouvelle rhétorique (Bruxelles : Editions de l'Université de Bruxelles)

Plantin, Christian. 2002 « Les topoï comme discours pivots », Eggs, Ekkehard (ed.). Topoi, discours, arguments (Beihefte der Zeitschrift für französische Sprache und Literatur, ZFSL) (Stuttgart : Steiner), $87-111$

Plantin, Christian. 2004b. « On the inseparability of emotion and reason in argumentation », Weigand, E. (ed.) Emotions in Dialogic Interactions (Amsterdam ; Benjamins), 265-276

Plantin, Christian. 2004a. « Sans démontrer ni (s')émouvoir », Meyer, Michel (éd.). Perelman - Le renouveau de la rhétorique (Paris: PUF), 65-80

Plantin, Christian. 2006a. « Ohne Demonstration und Emotion? », Kopperschmidt, Josef (ed.). Die Neue Rhetorik. Studien zu Chaim Perelman (München : Fink) [version allemande développée de «Sans démontrer ni s'émouvoir » 2004)], 281-295

Plantin, Christian. 2004b. « Ad passiones - Sur les affects de l'argumentation », Marques, Maria A. Pereira, Maria E. et al. (eds). Prácticas de Investigação em Análise Linguística do Discurso, (Braga : Universidade do Minho), 163-179 
Sitri, Frédérique. 2003. L'objet du débat. La construction des objets de discours dans des situations argumentatives orales (Paris: Presses de la Sorbonne Nouvelle)

Whately, Richard. 1844. Elements of logic. Including the substance of the article in the Encyclopedia Metropolitana. With additions, \& c. 8th edition, revised (London : Fellowes)

http://www.archive.org/details/elementsoflogic00whatuoft.

Wenzel, Joe W. 1987. « The rhetorical perspective on argument », Eemeren Frans H. van, Rob Grootendorst et al. (eds). 1987, Proceedings of the Conference on Argumentation 1986. 3 : Argumentation. Across the Lines of discipline (Dordrecht : Foris), 101-109

\section{NOTES}

1. Ce texte est la version remaniée d'un article publié en anglais dans Argumentation 2009, 2, «Perelman and Beyond. Form the Rethorical Tradition to Argumentation Studies », Amossy, Koren and Yanoshevsky (eds.).

2. sylva rhetoricæ, http://rhetoric.byu.edu/

3. L'expression « figure du discours » est parfois utilisée pour désigner la fallacie de «forme de l'expression", par exemple dans Krabbe (1998): "Who is afraid of figures of speech?» Argumentation 12, 2 : 281-294.

4. Conley restreint par la suite son analyse aux « resemblances between Perelman's treatment of loci and Renaissance "place-logics"» (id.)

5. L'idée d'une « rhétorique sans éloquence » vient d'E. Lévinas 1987.

6. La Bruyère 1951 [1688]. "Des ouvrages de l'esprit ». Les caractères ou les mœurs de ce siècle, Cuvres complètes. Texte établi et annoté par J. Benda (Paris: Gallimard). Cette œuvre s'inscrit dans la tradition des Caractères de Théophraste, dont La Bruyère a donné une traduction. On peut considérer que ce genre correspond à l'établissement des ethè des auditoires rhétoriques potentiels, cf. Aristote, Rhétorique $1388 \mathrm{~b} 31$ : » Etudions après cela les caractères (ethè), à savoir ce que sont les gens en fonction des passions (pathè), des dispositions (hexeis), des âges et des conditions de fortune. »

7. Nous utiliserons les mots latins, à la fois parce qu'ils font référence à la période de grande force de la rhétorique, et parce qu'ils produisent un effet de distanciation bienvenu.

8. A une période plus récente, la question a été plus particulièrement discutée, par exemple par Lempereur (1990) ; Klinkenberg $(1990,2001)$. Wenzel a consacré un paragraphe vengeur à la vision de la rhétorique proposée par la Rhétorique générale, qu'il juge « alarmante » (1987:103).

9. Et si c'est la forme simple qui est choisie, elle n'est telle que par une double surprise.

10. Ces remarques ne sous-entendent aucune critique du discours orné, ni une aversion de principe pour la recherche du vrai par le beau, comme le veut l'authentique définition de la poésie.

11. La notion de «master trope » vient de Kenneth Burke (1969 [1945]), qui qualifie ainsi la métaphore, la métonymie, la synecdoque et l'ironie.

12. Certaines de ces étiquettes renvoient en gros au même concept, selon qu'on le désigne par son nom grec, latin ou français.

13. Une figure d'emphase et de répitition.

14. Les listes et les considérations qui suivent, construites autour des $\S 41$ et $42 \mathrm{du} T A$, doivent être situées dans une perspective historique.

La réflexion théorique sur les figures, dans ces paragraphes essentiels du TA s'appuie sur des données- de rhétorique ancienne: Quintilien, Institutio; Rhétorique à Herennius; R. Volkman, Hermagoras, oder Elemente der Rhetorik; Longin, Traité du sublime, traduit par Boileau;- de 
rhétorique moderne, post-renaissance : Audomari Talei, c'est-à-dire Omer Talon, Rhetoricce, libri duo ; Baron, De la rhétorique ou de la composition oratoire et littéraire, 1849 ; Dumarsais, Des tropes... 1730 ; Vico, Delle instituzioni oratorie ; et enfin Chaignet, La rhétorique et son histoire, 1888, qui est la dernière rhétorique intégrale publiée en France avant la délégitimation de la rhétorique. Le seul auteur contemporain cité est Jean Paulhan, Les hain-tenys; Les figures ou la rhétorique décryptée. La première réélaboration du système des figures dans le cadre d'une théorie rhétorique intégrale est celle de H. Lausberg, Handbuch der literarischen Rhetorik, 1960.

15. Parmi eux, on compte en particulier, le type auquel appartient lecteur standard, relativement informé, des Rhétoriques: c'est ce qui rend plausible les propositions de ces ouvrages.

16. Le traité mentionne l'interpretatio parmi les figures de choix et de présence, ce qui se comprend bien dans le cadre d'une des définitions classiques du terme figure comme une figure de répétition, c'est-à-dire une structure de discours par laquelle un mot-clé d'une assertion est répété et spécifié par un quasi-synonyme (Ad. Her. IV : 38) ; c'est une forme de reformulation précisante. On comprend sans difficulté en quoi cette forme d'interpretatio marque le choix d'un éclairage et confère de la présence à l'être «interprété ». Mais l'exemple destiné à illustrer la notion d'interpretatio oriente a priori dans une direction toute différente, et on peut se demander si l'on n'a pas plutôt affaire à un enthymème plutôt qu'à une figure; un travail en cours est consacré à ce problème. Notons au passage que ce type de problème est assez caractéristique de la façon de faire du $T A$, où les thèses sont suivies d'exemples qui n'ont parfois rien de pédagogique, et ne se réfèrent à l'illustré que sous un point de vue très spécial. Quoi qu'il en soit, il reste que la distinction entre topos et trope est labile (voir Dyck 2003).

17. Libération (13 décembre $2007: 7$ ) fournit un très amusant exemple d'une discussion autour du processus de « choix » des données dans le débat sur le réchauffement climatique.

18. prosumptio et reprehensio sont deux formes de correctio (Lausberg 1960, §785. 3)

19. Il n'y a aucune incohérence à trouver l'interpretatio à la fois dans la rubrique des figures de choix et des figures de présence: le choix confère ipso facto de la présence, et des figures spécifiques viennent renforcer cette présence.

20. Le refus des émotions considérées comme systématiquement fallacieuses est une autre ligne de partage entre argumentation et rhétorique (Plantin 2004b).

21. Pour un essai de traitement des émotions dans le cadre de l'analyse du discours en général et de l'analyse de l'argumentation en particulier, voir Plantin 1998 et les publications correspondantes à l'adresse http://icar.univ-lyon2.fr/Membres/cplantin/Index.htm.

22. Le discours est un exemple d'objet naturel qui n'est pas susceptible de recevoir un traitement global unifié dans le cadre d'une grande théorie explicative. De tels objets complexes doivent être décomposés en plusieurs composantes relativement indépendantes et modélisables. L'ensemble de "composante + modèle" constitue un module. En principe, les modules ont des interfaces bien définis.

\section{RÉSUMÉS}

A partir du traitement des figures dans le Traité de l'Argumentation (TA) de Perelman et OlbrechtsTyteca, cet article porte sur la question de la place des figures dans la théorie de l'argumentation. La première partie revient brièvement sur différentes formes de rhétorique, dont une rhétorique 
de l'ornement ayant pour instrument principal les figures. Une théorie moderne de l'argumentation orientée vers la recherche des fallacies établit une coupure radicale entre argumentation, instrument de recherche de la vérité et rhétorique ornée, que Locke caractérise comme «a powerful instrument of error and deceit». Cette conception suppose l'usage d'un langage qu'on peut rapidement caractériser comme «artificiel», qui n'est pas celui de l'argumentation ordinaire. Avant d'être des «decorations » les figures sont des «instruments » de structuration de la parole; et les topoi, instruments de l'argumentation, n'obéissent pas à d'autres mécanismes que les tropes, qui semblent toujours parler à côté et manquer leur objet. La seconde partie s'intéresse à une partie quelque peu délaissée du TA, l'usage qu'il fait des figures et la vision théorique, à la fois cavalière et décisive, qu'il en propose. Le TA ouvre la voie à une conception des figures non pas comme "vain ornement " mais comme une première sémantique $\mathrm{du}$ discours argumentatif; c'est ce qu'on peut montrer sur le cas des «figures du choix, de la présence et de la communion ». En conséquence, la pensée des figures peut et doit être intégrée à une théorie de l'argumentation qui prend en compte le langage dans la parole, avec ses stratégies de construction des objets, du locuteur, de l'interlocuteur et de leurs émotions.

This paper deals with the treatment of figures of speech in Perelman's and Olbrechts-Tyteca's Treatise on Argumentation (TA), and, more broadly, with the place of figures in argumentation theory. The contrast between "a rhetoric of figures" and "a rhetoric of argument," which can be traced back to Ramus, was revived in the seventies by the perception of an incommensurability between TA and the École de Liège's "General Rhetoric". Modern theories of argumentation, oriented towards characterizing and denouncing fallacious discourse, emphasize the gap between sound argumentative discourse and discourse that is a "powerful instrument of error and deceit » (Locke). This concept of a gap envisions language as ideal / transparent - a revised language that is not the language of ordinary argumentation. In contrast, we argue that figures are not basically "decorative"; they are manifestations of the complex process of language structuring in speech. Thus rejecting figures amounts to a negation of discourse as such. We then turn to a somewhat neglected aspect of the $T A$, its both decisive and somewhat cavalier theory of figures of speech, and its extended use and re-definition of a complex set of figures. We argue that the $T A$, in its quest for descriptive adequacy, breaks with the traditional and comfortable concept of figures as useless fallacious "ornaments", and provides us with the first description of what could be characterized as the semantic level of ordinary argumentative discourse. This will be shown on the case of "figures of choice, presence and communion", and could be extended to the discursive construction of objects and participants, including the speaker and her emotions.

\section{INDEX}

Mots-clés : fallacie, figure, module discursifs, objet de discours, ornement, sémantique du discours

Keywords : discursive module, discursive object, fallacy, figure, ornament, semantic of discourse

\section{AUTEUR}

\section{CHRISTIAN PLANTIN}

CNRS, Université de Lyon 